\title{
Hemophagocytic Lymphohistiocytosis: manifestation of an untamed immune system
}

\author{
Narendra Kumar Bagri ${ }^{1}$ \\ Received: 12 May 2020 / Accepted: 12 May 2020 / Published online: 26 May 2020 \\ (C) Dr. K C Chaudhuri Foundation 2020
}

Hemophagocytic lymphohistiocytosis (HLH) is a hyperinflammatory state secondary to impaired cytotoxicity and resultant cytokine storm, presenting with fever, multi-organ failure, cytopenias, hyperferritinemia and coagulation abnormalities. HLH can either be primary/familial caused by mutations in FHLH (familial HLH) genes or secondary to various infections, malignancy, immunodeficiency or rheumatic diseases; in which it is widely known as macrophage activation syndrome (MAS). The impaired cytotoxicity and aberrant immune activation leading to cytokine storm is the common pathogenic mechanism for all forms of HLH [1]. HLH is a life threatening condition with mortality rates as high as $95 \%$ in FHLH and 20-30\% in secondary HLH. However, with timely diagnosis and the current immunosuppressive protocols, the mortality rates have optimistically reduced to $40 \%$ in FHL and less $10 \%$ for MAS $[1,2]$. Over the last decade there has been increased awareness about HLH but despite this it likely that only tip of the iceberg is being surfaced as many patients with unremitting fever, systemic inflammation, multi-organ involvement and hyperferritinemia may indeed be having underlying HLH [3].

In the current issue of this Journal, Nandhakumar et al. have published their experience with 52 Indian children with HLH [4]. In the present series, infection associated HLH constituted an important cause of HLH (73\%) with dengue being the most common inciting trigger; contrasting to the data from Taiwan where infection associated HLH accounted for $53 \%$ of all cases, whereas $24 \%$ were secondary to underlying rheumatic disease contrary to $9 \%$ in the present series [5]. These differences may be due to variability in referral patterns or the classification criteria used for identifying HLH. The recently proposed criteria including 2016 MAS classification criteria,

Narendra Kumar Bagri

drnarendrabagri@yahoo.co.in

1 Division of Pediatric Rheumatology, Department of Pediatrics, All India Institute of Medical Sciences, New Delhi, India
MAS/systemic juvenile idiopathic arthritis (SJIA) [MS] score with a sensitivity 73 and $85 \%$ and specificity of 99 and $95 \%$ respectively, seems more promising for early detection of MAS in underlying rheumatic disease specially, SJIA [6, 7]. Despite the availability of these classification tools for HLH/ MAS, its diagnosis is challenging and it is important to closely observe for evolving clinical symptoms and trend of laboratory parameters for contemplating the underlying diagnosis.

The therapeutic strategies opted by authors include use of various drugs ranging from intravenous immunoglobulins, corticosteroids to complete HLH 2004 protocol. It is intriguing to note that more than half of the children with secondary HLH and $65 \%$ of those with dengue, improved with symptomatic management and did not require immunosuppressive therapy. In the present study, etoposide/cyclosporine based $\mathrm{HLH}$ protocol has been used in 5 children with secondary HLH, however, the outcome of this subgroup is unclear. Although the authors have advised a useful algorithmic approach to guide therapeutic decisions, it would be prudent to chalk out the immunosuppressive plan in joint consultation with the oncologist and rheumatologist.

The authors have discussed the limitation of clinical and laboratory parameters in differentiating familial and secondary HLH and this emphasizes the need of genetic studies which are lacking in the present study, to clearly distinct these entities. However, getting genetic studies in all children with HLH may not be feasible in resource poor settings like ours. In this regard, the MAS/HLH [MH] score may help in differentiating MAS from FHLH [8] and guide in rationalising the genetic studies in our settings. This distinction is imperative owing to the therapeutic implications for FHLH, wherein timely referral for hematopoietic stem cell transplantation is vital for better outcome. Despite the retrospective design, this study shall increase awareness about HLH, in particular, secondary HLH and may act as a nidus for prospective trials for tailoring management strategies best suited for children in developing countries. 


\section{Compliance with Ethical Standards}

Conflict of Interest None.

\section{References}

1. Henderson LA, Cron RQ. Macrophage activation syndrome and secondary hemophagocytic lymphohistiocytosis in childhood inflammatory disorders: Diagnosis and management. Pediatr Drugs. 2020;22:29-44.

2. Simon AC, Delhi Kumar CG, Basu D, Ramesh Kumar R. Hemophagocytic lymphohistiocytosis in children: clinical profile and outcome. J Pediatr Hematol Oncol. 2020;1. https://doi.org/10. 1097/MPH.0000000000001791.

3. Crayne C, Cron RQ. Pediatric macrophage activation syndrome, recognizing the tip of the iceberg. Eur J Rheumatol. 2019;7:13-20. https://doi.org/10.5152/eurjrheum.2019.19150.

4. Nandhakumar D, Loganatha A, Sivasankaran M, Sivabalan S, Munirathnam D. Hemophagocytic lymphohistiocytosis in children. Indian J Pediatr. 2020 Feb 14. https://doi.org/10.1007/s12098-02003190-6.
5. Chen T-Y, Hsu M-H, Kuo H-C, Sheen J-M, Cheng M-C, Lin Y-J. Outcome analysis of pediatric hemophagocytic lymphohistiocytosis. J Formos Med Assoc. 2020. https://doi.org/10.1016/j.jfma.2020.03. 025 .

6. Ravelli A, Minoia F, Davì S, et al. 2016 classification criteria for macrophage activation syndrome complicating systemic juvenile idiopathic arthritis: A European league against rheumatism/American College of Rheumatology/Paediatric rheumatology international trials organisation collaborative initiative. Ann Rheum Dis. 2016;75: 481-9.

7. Minoia F, Bovis F, Davì S, et al. Development and initial validation of the MS score for diagnosis of macrophage activation syndrome in systemic juvenile idiopathic arthritis. Ann Rheum Dis. 2019;78: 1357-62.

8. Minoia F, Bovis F, Davì S, et al. Development and initial validation of the macrophage activation syndrome/primary hemophagocytic lymphohistiocytosis score, a diagnostic tool that differentiates primary hemophagocytic lymphohistiocytosis from macrophage activation syndrome. J Pediatr. 2017;189:72-8.e3.

Publisher's Note Springer Nature remains neutral with regard to jurisdictional claims in published maps and institutional affiliations. 